\title{
$p$-Version error estimation for linear elasticity
}

\author{
T. Pannachet $\cdot$ H. Askes $\cdot$ L. J. Sluys
}

Received: 19 November 2007 / Accepted: 8 August 2008 / Published online: 9 September 2008

(C) The Author(s) 2008. This article is published with open access at Springerlink.com

\begin{abstract}
An error estimator, formulated earlier for $h$-adaptive strategies, is extended for use in the $p$-version finite element analysis. The estimation of error is based on solving a series of local problems, based on patches consisting of elements surrounding each node, with prescribed homogeneous essential boundary conditions. Unlike the original approach in which a patch was constructed based on one element, each patch in the present scheme is automatically formed based on a number of elements surrounding a corresponding node. The present scheme, based on enhancing the degree of interpolation, provides a better estimate than the original $h$-scheme while still preserving the original lower bound property. The capability of the new scheme is investigated in some numerical examples in terms of its global and local performance.
\end{abstract}

Keywords Discretisation error - Error estimation . Hierarchical finite elements · Linear elasticity

\footnotetext{
T. Pannachet $(\varangle)$

Department of Civil Engineering, Khon Kaen University,

Khon Kaen, Thailand

e-mail: tanpan@kku.ac.th

H. Askes

Department of Civil and Structural Engineering,

University of Sheffield, Sheffield, UK

e-mail: h.askes@sheffield.ac.uk

L. J. Sluys

Faculty of Civil Engineering and Geosciences, Delft University of Technology, Delft, The Netherlands

e-mail: 1.j.sluys@tudelft.nl
}

\section{Introduction}

An important component of adaptive finite element analysis is how to assess the local error accurately. This error information normally gives a clue where and to which extent some parts of the mesh should be enhanced so that the finite element analysis can provide acceptably accurate and cost effective results. As such, the so-called a posteriori error estimators, which approximate the actual error at the end of the calculation, play an important role in ensuring reliability of finite element models. The error information, which is the focus of this research, refers to the error that is caused by inadequate discretisation of the finite element analysis, which is also known as the discretisation error.

Basically, a posteriori error estimators can be categorised in two main groups namely the recovery type (e.g., [20,21]) and the residual type (e.g., [5,7]). Here, the residual-type error estimators are employed. The methods, pioneered by the work of Babuška and Rheinboldt [5], determine the error by calculating the residual of the finite element solutions in each local space. Without relying on the superconvergence property ${ }^{1}$ of some sample points in the problem domain as in the recovery type, the residual-type error estimators can be applied to a wider variety of problems, including non-homogeneous higher-order interpolation or even nonlinear solution control. The methods, such as that of Babuška and Rheinboldt [5], determine the error by calculating the residual of the finite element solutions in each local space. However, the method is less robust and more computationally expensive than the recovery type since the calculation

\footnotetext{
${ }^{1}$ Superconvergence property belongs to some points where a very accurate solution can be obtained. They are usually the quadrature points [19].
} 
is complicated by solving the flux jump across the edge of the local domains.

Recently, the main shortcoming of the conventional residual type error estimation has been overcome by the introduction of a new residual type error estimator of Díez et al. [7]. The method approximates local errors without the necessity to compute the flux jump, thus the computational cost can be significantly diminished. The scheme can be easily integrated in a conventional finite element program and has been successfully applied to linear as well as nonlinear problems $[8,9,15]$. With such attractive benefits, the approach seems to be very promising and deserves a further development.

In this study, we apply a polynomial enrichment scheme (a so-called $p$-enrichment) to provide an alternative to the local $h$-refinement used in [7]. With a higher-order polynomial interpolation, the approximation can have much faster convergence to the exact solutions that contain the same order of polynomials, especially when the solution is rather smooth. Moreover, using a hierarchical extension of the shape functions for each local domain simplifies the error estimation process. Instead of subdividing each local domain, the reference solution is simply constructed by adding higherorder degrees of polynomials, also without changing any available stiffness matrix components. It is then of interest to study if the $p$-enrichment can also be applied in the context of error estimation, following the work of Díez et al. [7].

This article starts with a mathematical definition of the discretisation error in the finite element method, which is usually measured in terms of an energy norm. Then, we address some basic ideas about standard residual-type error estimation, which later leads to the formulation of a simple error estimator used in our research. The article ends with some investigations about performances and some critical comments about the proposed method.

\section{Discretisation error}

The discretisation error, $\boldsymbol{e}$, is defined as

$\boldsymbol{e}:=\boldsymbol{u}-\boldsymbol{u}_{(h, p)}$

i.e. the difference between the exact solution to the mathematical model, $\boldsymbol{u}$, and the finite element solution, $\boldsymbol{u}_{(h, p)} \in$ $\mathcal{V}_{(h, p)}$, where the finite element space $\mathcal{V}_{(h, p)} \subset \mathcal{V}$ and $\mathcal{V}:=$ $\left\{\boldsymbol{v} \in H^{1}(\Omega) ; \boldsymbol{v}=0\right.$ on $\left.\Gamma_{d}\right\}$. The solution is obtained by solving the set of equations

$\mathcal{B}\left(\boldsymbol{u}_{(h, p)}, \boldsymbol{v}_{(h, p)}\right)=\mathcal{F}\left(\boldsymbol{v}_{(h, p)}\right) \quad \forall \boldsymbol{v}_{(h, p)} \in \mathcal{V}_{(h, p)}$

where the terms $\mathcal{B}(\cdot, \cdot)$ and $\mathcal{F}(\cdot)$ denote a symmetric positivedefinite bilinear form and a linear form, respectively.

The error $\boldsymbol{e}$ in Eq. 1 cannot be computed directly since the exact solution $\boldsymbol{u}$ is generally unknown. Nevertheless, as a more refined/enriched discretisation gives a better approximation to the actual solution $\boldsymbol{u}$, we can closely represent the actual solution $\boldsymbol{u}$ by a very fine discretisation (so-called reference mesh), via $h$-extension and/or $p$-extension, for example. That is,

$\mathcal{B}\left(\boldsymbol{u}_{(\tilde{h}, \tilde{p})}, \boldsymbol{v}_{(\tilde{h}, \tilde{p})}\right)=\mathcal{F}\left(\boldsymbol{v}_{(\tilde{h}, \tilde{p})}\right) \quad \forall \boldsymbol{v}_{(\tilde{h}, \tilde{p})} \in \mathcal{V}_{(\tilde{h}, \tilde{p})}$

is to be solved and used as a close representative to the actual model. It should be noted that both $h$ and $p$ factors are not necessarily enhanced at the same time to form the reference solution. However, at least one factor needs to be upgraded to form the reference system of equations.

The finite element solution from the refined/enriched system $\boldsymbol{u}_{(\tilde{h}, \tilde{p})}$, is now denoted as a reference to the actual solution $\boldsymbol{u}$. As a consequence, the discretisation error, defined in Eq. 1, is approximated by

$\boldsymbol{e} \approx \boldsymbol{u}_{(\tilde{h}, \tilde{p})}-\boldsymbol{u}_{(h, p)}=: \boldsymbol{e}_{(\tilde{h}, \tilde{p})}$

The approximation involved in Eq. 4 is sufficiently accurate because the actual solution $\boldsymbol{u}$ is much closer to the solution from the refined system $\boldsymbol{u}_{(\tilde{h}, \tilde{p})}$ than to the primary solution $\boldsymbol{u}_{(h, p)}$.

In order to provide a proper measurement of global and elemental error, the discrete error should be measured in a well-defined norm. A classical option, also employed in this contribution, is the measurement of error in an energy norm defined as

$\|\boldsymbol{e}\|:=\sqrt{\mathcal{B}(\boldsymbol{e}, \boldsymbol{e})}=\sqrt{\sum_{k} \mathcal{B}_{k}(\boldsymbol{e}, \boldsymbol{e})}=\sqrt{\sum_{k}\|\boldsymbol{e}\|_{k}^{2}}$

where the subscript $k$ denotes the error contribution obtained from the elemental level. The global estimation is obtained by summing up the element contributions. The global error measure $\|\boldsymbol{e}\|$ is used in consideration whether or not the finite element solution is acceptably accurate. Furthermore, the elemental error measure of the element $k$,

$\|\boldsymbol{e}\|_{k}:=\sqrt{\mathcal{B}_{k}(\boldsymbol{e}, \boldsymbol{e})}$

is necessary in driving the mesh adaptive process.

\section{Standard residual-type error estimation}

The standard residual-type error estimation can be formulated either explicitly or implicitly. Whereas the explicit version employs the residuals in the current approximation directly, the implicit version uses the residuals indirectly via a set of local algebraic equations. Obviously, the implicit version, in comparison to the explicit version, requires more computational effort in solving an additional set of equations. The bigger effort, however, pays for the approximate error function, which is subsequently measured in a quantified norm. 


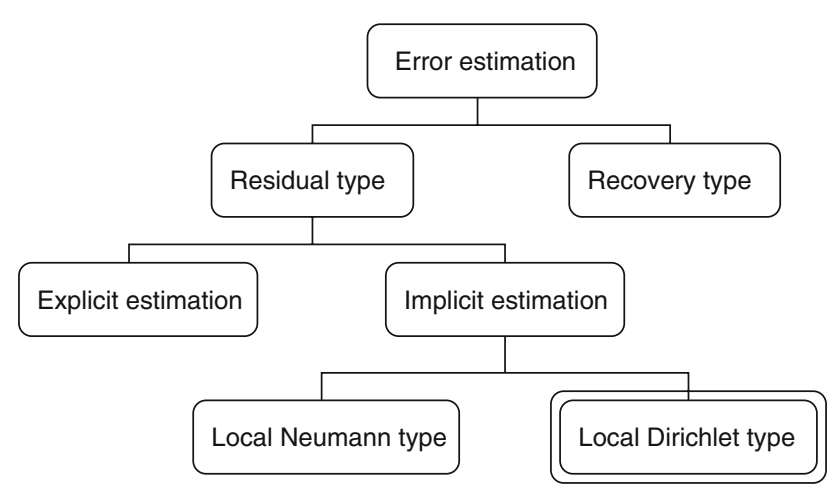

Fig. 1 Error assessment techniques in finite element analysis. The double-bounded box refers to the type used in this research

This error estimate provides more accurate information than obtained from the explicit version that relies on the inequality setting $[4,18]$. Figure 1 shows an overview of error assessment techniques used in finite element analysis.

In this research, we concentrate on the implicit error estimation. The method consists of three components, i.e.

- a set of error equations,

- a reference discretisation, and

- a local computational framework.

Basically, the set of error equations is formulated based on residuals in a global computational framework. Without the known exact solutions, the residuals are estimated by setting the reference discretisation via either $h$-extension, p-extension, or any other mesh improvement approach. Finally, the computational costs involved with the reference discretisation can be reduced importantly by replacing the solution of a global system by the solution of a series of local problems.

\subsection{Setting of error equations based on residuals}

The residual-type error estimator, as its name implies, approximates the error based on residuals, i.e. the amount by which the finite element solution fails to satisfy pointwise the equilibrium equation in the mathematical model. Using the difference between Eqs. 2, 3, and 4, leads to a set of error equations

$$
\begin{aligned}
\mathcal{B}\left(\boldsymbol{e}_{(\tilde{h}, \tilde{p})}, \boldsymbol{v}_{(\tilde{h}, \tilde{p})}\right)= & \mathcal{R}^{u}\left(\boldsymbol{v}_{(\tilde{h}, \tilde{p})}\right) \\
= & \mathcal{F}\left(\boldsymbol{v}_{(\tilde{h}, \tilde{p})}\right)-\mathcal{B}\left(\boldsymbol{u}_{(h, p)}, \boldsymbol{v}_{(\tilde{h}, \tilde{p})}\right) \\
& \forall \boldsymbol{v}_{(\tilde{h}, \tilde{p})} \in \mathcal{V}_{(\tilde{h}, \tilde{p})}
\end{aligned}
$$

with the boundary condition that $\boldsymbol{e}=\mathbf{0}$ on $\Gamma_{d}$. The residual $\mathcal{R}^{u}$, which is based on the primary unknown $\boldsymbol{u}$, can be interpreted as a fictitious load by which the approximate solution deviates from the actual solution.

\subsection{Setting of local computational framework}

In fact, one can estimate the error of a finite element model by comparing the finite element solutions obtained from the original mesh to those from the enhanced mesh, by which the results could be compared pointwise rather than through a single scalar such as the energy norm. Unfortunately, this would require a large amount of computations involved with the finer mesh. There is obviously little value in estimating the error of a coarse discretisation by solving a global system of equations according to an enhanced discretisation. The computational costs involved with the error estimation would far outweigh those involved with solving for $\boldsymbol{u}_{(h, p)}$, while at the same time an improved solution $\boldsymbol{u}_{(\tilde{h}, \tilde{p})}$ is already provided. By virtue of $\boldsymbol{u}_{(\tilde{h}, \tilde{p})}$, the solution $\boldsymbol{u}_{(h, p)}$ has become redundant, and so has $\boldsymbol{e}_{(\tilde{h}, \tilde{p})}$. In contrast, an efficient calculation of $\boldsymbol{e}_{(\tilde{h}, \tilde{p})}$ should involve local (rather than global) solutions of $\boldsymbol{u}_{(\tilde{h}, \tilde{p})}$.

Since Eq. 7 is defined globally, it requires a large amount of computer resources. In order to avoid this, the local spaces $\mathcal{V}_{k}, k=1,2, \ldots, n$ and $\mathcal{V}_{k} \subset \mathcal{V}$, are defined and the residualbased error is computed in each local space. That is, instead of solving Eq. 7, we solve a set of local equations

$$
\mathcal{B}_{k}\left(\boldsymbol{e}_{(\tilde{h}, \tilde{p})}, \boldsymbol{v}_{(\tilde{h}, \tilde{p})}\right)=\mathcal{R}_{k}^{u}\left(\boldsymbol{v}_{(\tilde{h}, \tilde{p})}\right) \quad \forall \boldsymbol{v}_{(\tilde{h}, \tilde{p})} \in \mathcal{V}_{k(\tilde{h}, \tilde{p})}
$$

where the local residual is defined as

$$
\begin{array}{r}
\mathcal{R}_{k}^{u}\left(\boldsymbol{v}_{(\tilde{h}, \tilde{p})}\right)=\mathcal{F}_{k}\left(\boldsymbol{v}_{(\tilde{h}, \tilde{p})}\right)-\mathcal{B}_{k}\left(\boldsymbol{u}_{(h, p)}, \boldsymbol{v}_{(\tilde{h}, \tilde{p})}\right)+\cdots \\
\ldots \int_{\partial \Omega_{k} \backslash\left(\partial \Omega_{k} \cap \Gamma_{n}\right)} \frac{\partial \boldsymbol{u}}{\partial \boldsymbol{n}_{k}} \boldsymbol{v}_{(\tilde{h}, \tilde{p})} \mathrm{d} \Gamma
\end{array}
$$

As a result from the integration by parts on each local domain, the additional contribution, which is the last term in the right-hand side of Eq. 9, represents the normal derivatives (or flux) on the interelement boundary $\partial \Omega_{k}$ as well as on $\Gamma_{d}$ and cancels in the global system of equations (cf. Eq. 7). Note that the contribution of the normal derivative on element edges on $\Gamma_{n}$, i.e. $\int_{\partial \Omega_{k} \cap \Gamma_{n}} \frac{\partial \boldsymbol{u}}{\partial \boldsymbol{n}_{k}} \boldsymbol{v} \mathrm{d} \Gamma$ is included in $\mathcal{F}_{k}(\boldsymbol{v})$ as defined earlier.

To obtain the error associated with the primary unknowns $\boldsymbol{u}_{(h, p)}$, the local error equations (cf. Eq. 8) must be solved. It is necessary to define a proper set of boundary conditions of these local problems.

\section{Boundary conditions of the local error equations}

A key ingredient in solving local error equations is setting the boundary conditions to be prescribed in Eq. 8. Taken from the global finite element setting, the only Dirichlet boundary condition defined in each local space $\Omega_{k} \subset \Omega$ is

$\boldsymbol{e}=\mathbf{0} \quad$ on $\quad \partial \Omega_{k} \cap \Gamma_{d}$ 
This is because the primary unknown $\boldsymbol{u}$ is exactly prescribed on the Dirichlet boundary $\Gamma_{d}$. Obviously, additional boundary conditions for the local problems are needed.

Basically, there are two subclasses of the implicit residual error estimation, depending on how the boundary conditions are defined in the local problems, namely the Neumann-type error estimation and the Dirichlet-type error estimation. See also the overview in Fig. 1.

\subsection{Local Neumann conditions}

The imposition of non-homogeneous flux boundary conditions (local Neumann conditions), represented by the last term of Eq. 9, may be set via the simple flux averaging technique as

$$
\begin{aligned}
& \frac{\partial \boldsymbol{u}_{(h, p)}}{\partial \boldsymbol{n}_{k}} \approx \frac{1}{2} \boldsymbol{n}_{k} \cdot\left\{\left(\nabla \boldsymbol{u}_{(h, p)}\right)_{k}+\left(\nabla \boldsymbol{u}_{(h, p)}\right)_{k^{\prime}}\right\} \\
& \text { on } \partial \Omega_{k} \cap \partial \Omega_{k^{\prime}}
\end{aligned}
$$

The considered edge of an element $k$ is shared by another (adjacent) element denoted as $k^{\prime}$. The introduction of two distinct indices $k$ and $k^{\prime}$ allows to describe jumps of the normal fluxes at the interelement boundary.

The simple averaging has been criticised for being ad-hoc and fails to respect the basic requirement for the local problem to be well-posed. Some researchers $[3,10]$ have proposed a new modification, the so-called equilibrated flux approach, by setting the equilibration condition

$$
\mathcal{F}_{k}(\boldsymbol{v})-\mathcal{B}_{k}\left(\boldsymbol{u}_{(h, p)}, \boldsymbol{v}\right)+\int_{\partial \Omega_{k} \backslash\left(\partial \Omega_{k} \cap \Gamma_{n}\right)} \frac{\partial \boldsymbol{u}}{\partial \boldsymbol{n}_{k}} \boldsymbol{v} \mathrm{d} \Gamma=0
$$

where $\boldsymbol{v}=1$ and $\boldsymbol{v}=\boldsymbol{\phi}$ (the interpolation function) are selected for zeroth-order equilibration and first-order equilibration conditions, respectively. And with the consistency condition

$$
\frac{\partial \boldsymbol{u}}{\partial \boldsymbol{n}_{k}}+\frac{\partial \boldsymbol{u}}{\partial \boldsymbol{n}_{k^{\prime}}}=\mathbf{0} \quad \text { on } \quad \partial \Omega_{k} \cap \partial \Omega_{k^{\prime}}
$$

the error equations are well-posed on the regular subspace and the resulting error estimator will provide a guaranteed upper bound of the exact error.

It should be noted that imposing only Neumann boundary conditions in the local problems is not sufficient. It is necessary to impose a proper set of Dirichlet conditions to eliminate the zero energy modes (rigid body modes), leading to solvability of the equations. Obviously, the Dirichlet conditions described in Eq. 10 are not sufficient for solving the local problems that are not attached to the Dirichlet boundary. To overcome this problem, one may reformulate the local problem over a reduced subspace where the zero energy modes have been factored out $[1,2,6]$.

\subsection{Local Dirichlet conditions}

Modelling of the equilibrated residual fluxes at the interelement boundaries generally requires high computational effort. To avoid such complicated computation, the local Neumann boundary conditions in conventional element residual method may be replaced by a set of local Dirichlet conditions. The method approximates local errors without the necessity to compute the flux jump, thus the computational cost can be significantly diminished. However, this assumption leads to a lower bound estimate that is often not of a good quality.

An improvement of the approach has been proposed by Díez et al. [7]. In their approach, an additional set of local error equations is introduced to help improving quality of the error estimate computed based on the elemental basis. The error function can be approximated by solving a set of local problems whose spaces overlap. These local (patch) spaces $\Omega^{\text {pat }}$ must be selected in such a way that

$-\bar{\Omega}=\bigcup_{k} \bar{\Omega}_{k}^{\text {pat }}$, i.e. all patches together cover the whole problem domain, and

- $\Omega_{i}^{\text {pat }} \cap \Omega_{j}^{\text {pat }} \neq \varnothing$, i.e. a patch $\Omega_{i}^{\text {pat }}$ must overlap partly at least one other patch $\Omega_{j}^{\text {pat }}$ that is in the neighbourhood. The overlapping of patches depends on how the reference mesh is chosen.

For a local space (or patch) $\Omega_{k}^{\text {pat }}$, a set of homogeneous boundary conditions is defined by suppressing error components as zero on the local boundary $\partial \Omega_{k}^{\text {pat }} \backslash\left(\partial \Omega_{k}^{\text {pat }} \cap \Gamma_{n}\right)$. The error estimate can then be obtained by finding $\xi \in \mathcal{V}_{k}^{*}$ where $\mathcal{V}_{k}^{*}:=\left\{\boldsymbol{v} \in H^{1}\left(\Omega_{k}^{\mathrm{pat}}\right) ; \boldsymbol{\xi}=\mathbf{0}\right.$ on $\left.\Gamma_{d} \cup \partial \Omega_{k}^{\text {pat }}\right\}$ from

$$
\begin{aligned}
\mathcal{B}_{k}\left(\boldsymbol{\xi}_{(\tilde{h}, \tilde{p})}, \boldsymbol{v}_{(\tilde{h}, \tilde{p})}\right)= & \mathcal{R}_{k}^{u}\left(\boldsymbol{v}_{(\tilde{h}, \tilde{p})}\right) \\
= & \mathcal{F}_{k}\left(\boldsymbol{v}_{(\tilde{h}, \tilde{p})}\right)-\mathcal{B}_{k}\left(\boldsymbol{u}_{(h, p)}, \boldsymbol{v}_{(\tilde{h}, \tilde{p})}\right) \\
& \forall \boldsymbol{v}_{(\tilde{h}, \tilde{p})} \in \mathcal{V}_{k(\tilde{h}, \tilde{p})}
\end{aligned}
$$

temporarily neglecting the last term appearing in Eq. 9. The space $\mathcal{V}_{k}=\operatorname{supp}\left(\Omega_{k}^{\mathrm{pat}}\right)$, thus $\mathcal{V}_{k} \subset \mathcal{V}$. In the original work [7], this first estimate $\boldsymbol{\xi}$ to $\boldsymbol{e}_{(\tilde{h}, \tilde{p})}$ is computed elementwise (i.e. the local space is based on one element) and denoted as the interior estimate.

Since the estimated error is suppressed to zero on the inter-patch boundaries, the obtained error solution is a poor approximation to the exact error. It is then necessary to enrich the first patch solutions by a set of patches overlapping the local space. Let $\Lambda_{l}$ be the local space that overlaps $\Omega_{k}$, find another error estimate $\eta \in \mathcal{U}_{l}^{*}$, where $\mathcal{U}_{l}^{*}:=\{\boldsymbol{v} \in$ $H^{1}\left(\Lambda_{l}^{\mathrm{pat}}\right) ; \boldsymbol{\eta}=\mathbf{0}$ on $\left.\Gamma_{d} \cup \partial \Lambda_{l}^{\mathrm{pat}}\right\}$ from another boundary value problem 


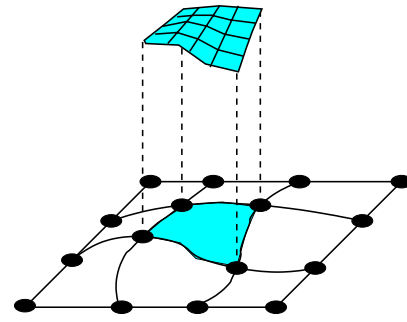

(I) Internal residual estimate

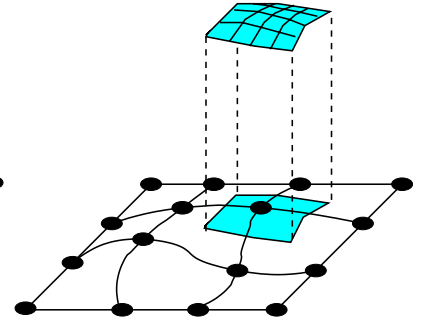

(2) Boundary jump recovery
Fig. 2 Two steps of residual-based error computation in the local Dirichlet-type framework based on local $h$-refinement [7]

$$
\begin{aligned}
\mathcal{B}_{l}\left(\boldsymbol{\eta}_{(\tilde{h}, \tilde{p})}, \boldsymbol{v}_{(\tilde{h}, \tilde{p})}\right)= & \mathcal{R}_{l}^{u}\left(\boldsymbol{v}_{(\tilde{h}, \tilde{p})}\right) \\
= & \mathcal{F}_{l}\left(\boldsymbol{v}_{(\tilde{h}, \tilde{p})}\right)-\mathcal{B}_{l}\left(\boldsymbol{u}_{(h, p)}, \boldsymbol{v}_{(\tilde{h}, \tilde{p})}\right) \\
& \forall \boldsymbol{v}_{(\tilde{h}, \tilde{p})} \in \mathcal{U}_{l}(\tilde{h}, \tilde{p})
\end{aligned}
$$

The second estimate $\eta$ is based on the collection of parts in surrounding elements to form each patch overlapping the element domain (thus interior domain) and is called the patch estimate [7]. Similar to the interior estimate $\xi$, the local errors on $\partial \Lambda_{l} \backslash\left(\partial \Lambda_{l} \cap \Gamma_{n}\right)$ are prescribed to zero. This patch estimate provides information of the error caused by the residual fluxes on the elemental boundaries. See Fig. 2 for an illustration of the two-step error computation.

To combine components from different patches, the contributions $\boldsymbol{\xi}$ and $\boldsymbol{\eta}$ must be adjusted to satisfy the Galerkin orthogonality property, that is

$\mathcal{B}_{l}\left(\boldsymbol{\xi}_{(\tilde{h}, \tilde{p})}, \boldsymbol{\eta}_{(\tilde{h}, \tilde{p})}\right)=0$ on $\Lambda_{l}$

Retrieving the interior estimate which is projected onto $\Lambda_{l}$, the patch solution in each $\Lambda_{l}$ can be recalculated as

$\eta_{(\tilde{h}, \tilde{p})}^{*}=\boldsymbol{\eta}_{(\tilde{h}, \tilde{p})}-\frac{\mathcal{B}_{l}\left(\boldsymbol{\eta}_{(\tilde{h}, \tilde{p})}, \boldsymbol{\xi}_{(\tilde{h}, \tilde{p})}^{*}\right)}{\mathcal{B}_{l}\left(\boldsymbol{\xi}_{(\tilde{h}, \tilde{p})}^{*}, \boldsymbol{\xi}_{(\tilde{h}, \tilde{p})}^{*}\right)} \quad \boldsymbol{\xi}_{(\tilde{h}, \tilde{p})}^{*} \quad$ in $\Omega_{l}$

where $\xi^{*}$ is the projection of the interior estimate $\boldsymbol{\xi}$ on $\Lambda_{l}$ which is adjusted as zero on $\partial \Lambda_{l} \backslash\left(\partial \Lambda_{l} \cap \Gamma_{n}\right)$ by

$\mathcal{B}_{l}^{*}\left(\boldsymbol{\xi}_{(\tilde{h}, \tilde{p})}^{*}, \boldsymbol{v}_{(\tilde{h}, \tilde{p})}\right)=\mathcal{B}_{l}\left(\boldsymbol{\xi}_{(\tilde{h}, \tilde{p})}, \boldsymbol{v}_{(\tilde{h}, \tilde{p})}\right) \quad \forall \boldsymbol{v}_{(\tilde{h}, \tilde{p})} \in \mathcal{U}_{l(\tilde{h}, \tilde{p})}$

where $\mathcal{B}_{l}^{*}(\cdot, \cdot)$ represents $\mathcal{B}_{l}(\cdot, \cdot)$ with the prescription of zero error on $\partial \Lambda_{l} \backslash\left(\partial \Lambda_{l} \cap \Gamma_{n}\right)$. The use of $\xi^{*}$ instead of $\xi$ in Eq. 17 ensures continuity of the estimated error function after setting the orthogonality to the interior values, which subsequently guarantees the continuity of the complete solution. It should be noted that the orthogonality setting procedure unfortunately causes some blind points on the Neumann's boundary where two patches meet. At those points on $\Gamma_{n}$, the estimation of zero error is obtained.

By adding the two components, the complete estimate becomes

$\boldsymbol{e}_{(\tilde{h}, \tilde{p})} \approx \boldsymbol{\xi}_{(\tilde{h}, \tilde{p})}+\eta_{(\tilde{h}, \tilde{p})}^{*}$
(A)

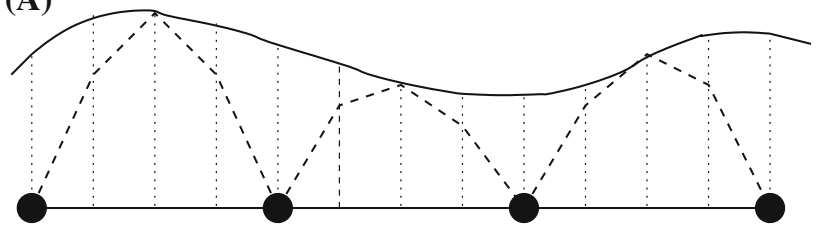

(B)

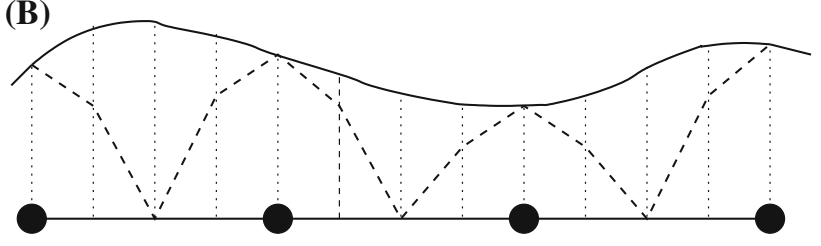

(C)

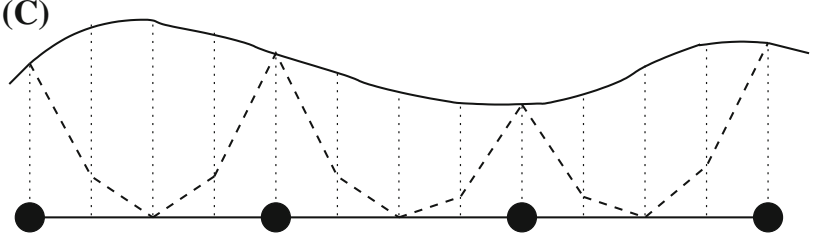

Fig. 3 The local Dirichlet method: a interior estimation $\xi$, b patch estimation before orthogonality setting $\eta$ and $\mathbf{c}$ patch estimation after the orthogonality setting $\eta^{*}$, after which contributions $\xi$ and $\eta^{*}$ are added. The exact errors and the estimated errors are shown in solid and dashed lines, respectively. The filled circles denote nodal points in the one-dimensional problem domain

by which non-zero errors can be obtained along the interpatch boundaries.

The estimated error can be measured in the energy norm by

$\left\|\boldsymbol{e}_{(\tilde{h}, \tilde{p})}\right\|_{k}^{2} \approx\left\|\boldsymbol{\xi}_{(\tilde{h}, \tilde{p})}\right\|_{k}^{2}+\left\|\boldsymbol{\eta}_{(\tilde{h}, \tilde{p})}^{*}\right\|_{k}^{2}$

and summed up to obtain the global error in the energy norm (cf. Eq. 5). Figure 3 illustrates how this local Dirichlet error estimation works in a one-dimensional setting. The method can be implemented following the flow chart in Table 1.

\section{Error estimation for non-uniform interpolation}

In the original version [7], two sets of local problems are formulated. First, the interior residual-based error is computed by setting a zero error on the edge of the elements which do not belong to the Neumann's boundary. The second set is to recover the residual on the boundary of the element by setting the patches, based on nodes or edges, overlapping the neighbouring elements.

Originally, the local Dirichlet type error estimator was formulated and successfully applied with a local $h$-extension. ${ }^{2}$

\footnotetext{
${ }^{2}$ Note that $h$-version and $p$-version refer here to the error estimation procedure, not to a possible global enhancement of the discretisation that may follow the error estimation.
} 
Table 1 Flow diagram for the error estimation [7]

For each patch of elements

1. Retrieve elements in the patch:

(a) subdivide the patch/enrich the shape functions,

(b) compute the refined/enriched stiffness matrix $\boldsymbol{K}_{(\tilde{h}, \tilde{p})}^{\mathrm{el}}:=\mathcal{B}_{k}(\boldsymbol{v}, \boldsymbol{v})$,

(c) compute the refined/enriched load vector $f_{(\tilde{h}, \tilde{p})}^{\mathrm{el}}:=$ $\mathcal{F}_{k}(\boldsymbol{v})$,

(d) interpolate solution vectors to the refined/ enriched system $\boldsymbol{u}_{\text {interp }}^{\text {el }}$, and

(e) add to the patch stiffness matrix $\boldsymbol{K}_{(\tilde{h}, \tilde{p})}^{\text {pat }}$, patch load vector $\boldsymbol{f}_{(\tilde{h}, \tilde{p})}^{\text {pat }}$ and patch solution vector $\boldsymbol{u}_{\text {interp }}^{\text {pat }}$.

2. Impose the error boundary conditions for the patch problem, i.e. $\boldsymbol{e}^{\text {pat }}=\mathbf{0}$ on $\partial \Omega^{\text {pat }} \backslash\left(\Omega^{\text {pat }} \cup \Gamma_{n}\right)$.

3. Solve for patch error vector $\boldsymbol{e}^{\text {pat }}$.

4. Retrieve existing patch error vector $\boldsymbol{e}^{\text {pat }, 0}$.

5. Set the orthogonality of the existing error vector and the new error vector $\boldsymbol{e}_{\text {orth }}^{\text {pat }}$.

6. Add the orthogonal new error vector to the global numbe$\operatorname{ring} \boldsymbol{e}^{\mathrm{glob}, 0}=\boldsymbol{e}^{\mathrm{glob}, 0}+\boldsymbol{e}_{\mathrm{orth}}^{\mathrm{pat}}$.

7. Continue to next patch.
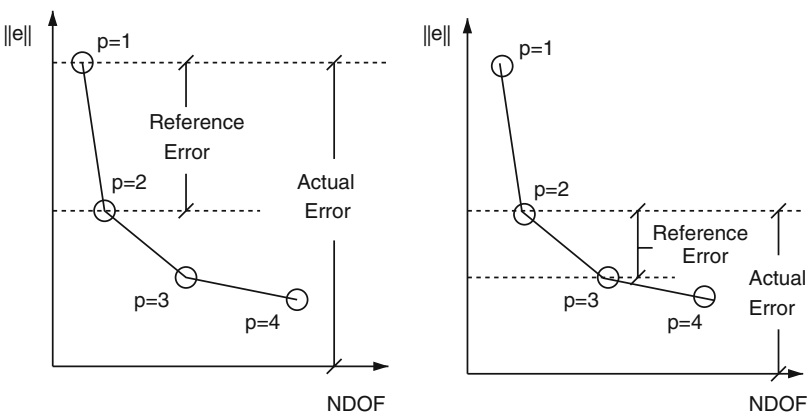

Fig. 4 Reference error and actual error in the local $p$-enrichment scheme: the reference for linear elements (left) and for quadratic elements (right)

To provide a proper reference discretisation in the case of non-uniform interpolation, a $p$-extension is more suitable. Instead of subdividing each local space (element), the degree of polynomial interpolation $(p)$ is upgraded to one higher order $(p+1)$. The error with respect to the reference mesh, in comparison to the actual error, is illustrated in Fig. 4.

We have implemented two error estimators for use with non-uniform interpolation, i.e.

- based on the element-based hierarchical enhancement [16], and

- based on the node-based hierarchical enhancement [17].

We have found that, although the node-based approach [12] facilitates the implementation, it requires a tedious
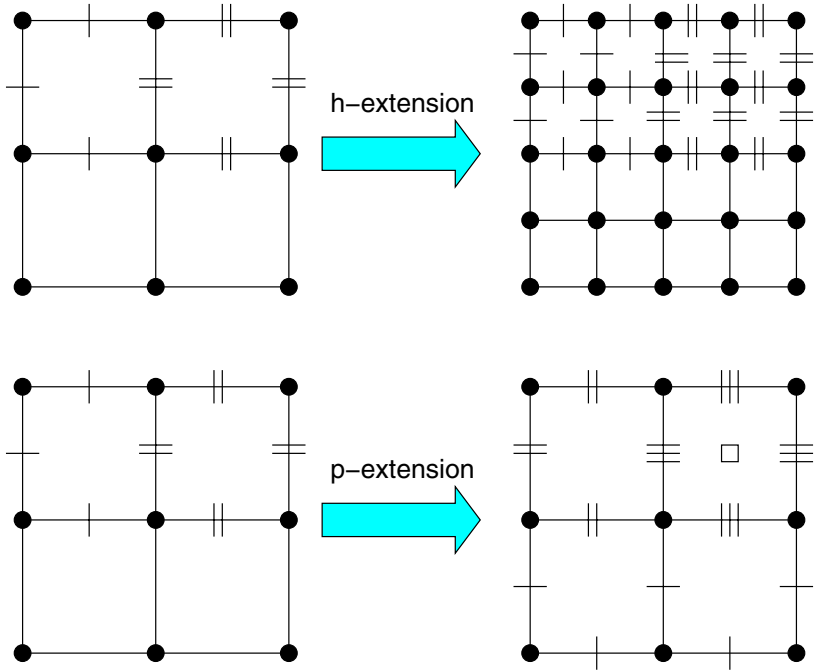

Fig. 5 Reference mesh in the framework of $h$ - and $p$-extensions for the problem with non-uniform higher-order elements. The short lines on element edges denote the edge modes and the square symbol denotes the internal mode

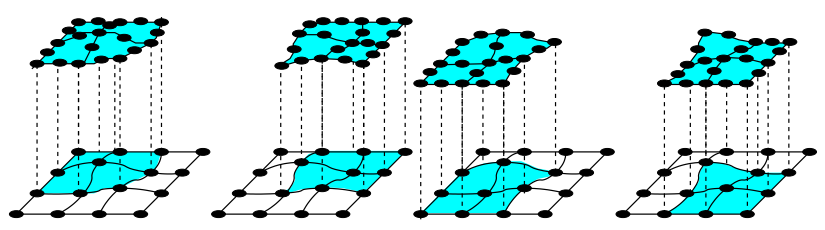

Fig. 6 Node-based patches for error estimation based on local penrichment

element selection procedure to avoid linear dependency problems usually found in the local model containing a small number of elements. As a consequence, this may diminish the robustness of the nodal $p$-enrichment method since the required minimum number of elements may keep growing if the polynomial order grows (see more details in [11, Appendix A]). The element-based approach [13], however, does not show such a shortcoming. For this reason, our $p$-version error estimation will only be based on the elementbased $p$-extension.

One problem remains. Unlike the $h$-version error estimation [7], it is impossible to use a portion of a certain finite element within a patch. Instead, the whole element must be taken. Automatically set based on each node, a patch can be constructed by a set of surrounding elements, as illustrated in Figs. 5 and 6. By this scheme, the global number of patches is fixed by the number of nodes. Each element is enriched by the number of patches that corresponds to the element type. For instance, contributions from three patches are combined to recover errors in a linear triangular element and contributions from four patches are used for a bilinear quadrilateral element. 


\section{Some implementational aspects}

\subsection{Solution mapping}

Discretising the continuous test function $\boldsymbol{v}$ with the finite element shape functions $\phi$, the error can be obtained by solving the local discretised equations

$$
\mathcal{B}_{k}\left(\boldsymbol{e}_{(\tilde{h}, \tilde{p})}, \boldsymbol{\phi}_{(\tilde{h}, \tilde{p})}\right)=\mathcal{F}_{k}\left(\boldsymbol{\phi}_{(\tilde{h}, \tilde{p})}\right)-\mathcal{B}_{k}\left(\boldsymbol{u}_{(h, p)}, \boldsymbol{\phi}_{(\tilde{h}, \tilde{p})}\right)
$$

which in the usual matrix-vector format reads

$\boldsymbol{K}_{(\tilde{h}, \tilde{p})}^{(k)} \mathbf{e}_{(\tilde{h}, \tilde{p})}^{(k)}=\boldsymbol{f}_{(\tilde{h}, \tilde{p})}^{(k)}-\check{\boldsymbol{K}}_{(\tilde{h}, \tilde{p})}^{(k)} \boldsymbol{a}_{(h, p)}^{(k)}$

where $\boldsymbol{a}$ are the nodal displacements,

$$
\begin{aligned}
\boldsymbol{K}_{(\tilde{h}, \tilde{p})}^{(k)} & :=\mathcal{B}_{k}\left(\boldsymbol{\phi}_{(\tilde{h}, \tilde{p})}, \boldsymbol{\phi}_{(\tilde{h}, \tilde{p})}\right) \\
& :=\int_{\Omega_{k}}\left(\nabla \boldsymbol{\phi}_{(\tilde{h}, \tilde{p})}\right): \boldsymbol{D}:\left(\nabla \boldsymbol{\phi}_{(\tilde{h}, \tilde{p})}\right) \mathrm{d} \Omega_{k}
\end{aligned}
$$

is the stiffness matrix,

$$
\begin{aligned}
\boldsymbol{f}_{(\tilde{h}, \tilde{p})}^{(k)} & :=\mathcal{F}_{k}\left(\boldsymbol{\phi}_{(\tilde{h}, \tilde{p})}\right) \\
& :=\int_{\Omega_{k}} \boldsymbol{\phi}_{(\tilde{h}, \tilde{p})} \cdot \boldsymbol{q} \mathrm{d} \Omega_{k}+\int_{\Gamma_{n} \cap \partial \Omega_{k}} \boldsymbol{\phi}_{(\tilde{h}, \tilde{p})} \cdot \boldsymbol{g} \mathrm{d} \Omega_{k}
\end{aligned}
$$

is the external force vector, and

$$
\begin{aligned}
\check{\boldsymbol{K}}_{(\tilde{h}, \tilde{p})}^{(k)} & :=\mathcal{B}_{k}\left(\boldsymbol{\phi}_{(h, p)}, \boldsymbol{\phi}_{(\tilde{h}, \tilde{p})}\right) \\
& :=\int_{\Omega_{k}}\left(\nabla \boldsymbol{\phi}_{(\tilde{h}, \tilde{p})}\right): \boldsymbol{D}:\left(\nabla \boldsymbol{\phi}_{(h, p)}\right) \mathrm{d} \Omega_{k}
\end{aligned}
$$

The last term in Eq. 22 indicates that the solution of the coarse mesh, $\boldsymbol{a}_{(h, p)}$, is mapped onto the refined mesh.

Alternatively, to make use of the existing stiffness matrix $\boldsymbol{K}$ for error computation, one can use an equivalent form as

$\boldsymbol{K}_{(\tilde{h}, \tilde{p})}^{(k)} \mathbf{e}_{(\tilde{h}, \tilde{p})}^{(k)}=\boldsymbol{f}_{(\tilde{h}, \tilde{p})}^{(k)}-\boldsymbol{K}_{(\tilde{h}, \tilde{p})}^{(k)} \check{\boldsymbol{a}}_{(\tilde{h}, \tilde{p})}^{(k)}$

where $\check{\boldsymbol{a}}_{(\tilde{h}, \tilde{p})}^{(k)}$ is interpolated using the original shape functions $\boldsymbol{\phi}_{(h, p)}$ that correspond to the new set of nodal positions $\boldsymbol{x}_{(\tilde{h}, \tilde{p})}$ in the enriched local problem, yielding

$\check{\boldsymbol{u}}_{(\tilde{h}, \tilde{p})}^{(k)}:=\boldsymbol{\phi}_{(h, p)}\left(\boldsymbol{x}_{(\tilde{h}, \tilde{p})}\right) \cdot \boldsymbol{a}_{(h, p)}^{(k)}$

The form in Eq. 26 is more efficient than that in Eq. 22 in terms of data storage. Also, computation of another integral for a modified stiffness matrix costs more than interpolation of the displacement field $\boldsymbol{u}$. However, the form in Eq. 22 provides more flexibility in selecting the reference mesh. For the hierarchical approach, use of the form in Eq. 26 is natural. This is because $\mathcal{V}_{(h, p)} \in \mathcal{V}_{(\tilde{h}, \tilde{p})}$, i.e. the existing shape functions are preserved and completely separate themselves from the set of additional degrees of freedom. Therefore, at the additional degrees of freedom, a zero contribution is simply
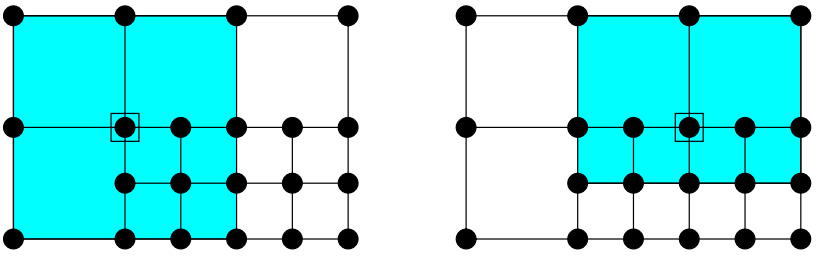

Fig. 7 Examples of patch selection in case of hanging nodes

added to form the interpolated set of the primary unknowns $\check{\boldsymbol{a}}_{(\tilde{h}, \tilde{p})}^{(k)}$.

\subsection{Irregular element connectivity}

The requirement of overlapping patches may complicate the patch selection process. An example is when hanging nodes exist. In such a case, the finite element interpolation requires special constraints at the hanging nodes so that the shape functions over the irregular partitioning are compatible.

It is obvious in the original $h$-version, where a patch consists of a part of each element in the neighbourhood, that it is merely impossible to obtain the patching scheme described in Fig. 2. However, as an alternative, the patches which constitute all neighbouring elements (as in the $p$-version, cf. Fig. 6) may be selected to include all involved parent-child relations (cf. Fig. 7), i.e. the local problems have to allow the element connecting to the hanging node to be in the part as well.

\section{Performance analyses}

\subsection{One-dimensional problem}

The first numerical example is a one-dimensional problem described by the ordinary differential equation

$-\frac{d^{2} u}{d x^{2}}(x)=6 x^{2}-3 x$

in $\Omega=] 0,1[$ and the Dirichlet boundary conditions are prescribed as $u(0)=u(1)=0$. The analytical solution for this problem is

$u(x)=\frac{\left(x^{3}-x^{4}\right)}{2}$

In order to investigate the performance of the error estimator, we start with measuring the capability of the local $p$-version in estimating the error in linear elements, in comparison with the local $h$-version. In this problem, each patch is selected based on one element, and, for the same reason as in [7], we assume that there exist only interior residuals due to the superconvergence property of the problem. Thus, 

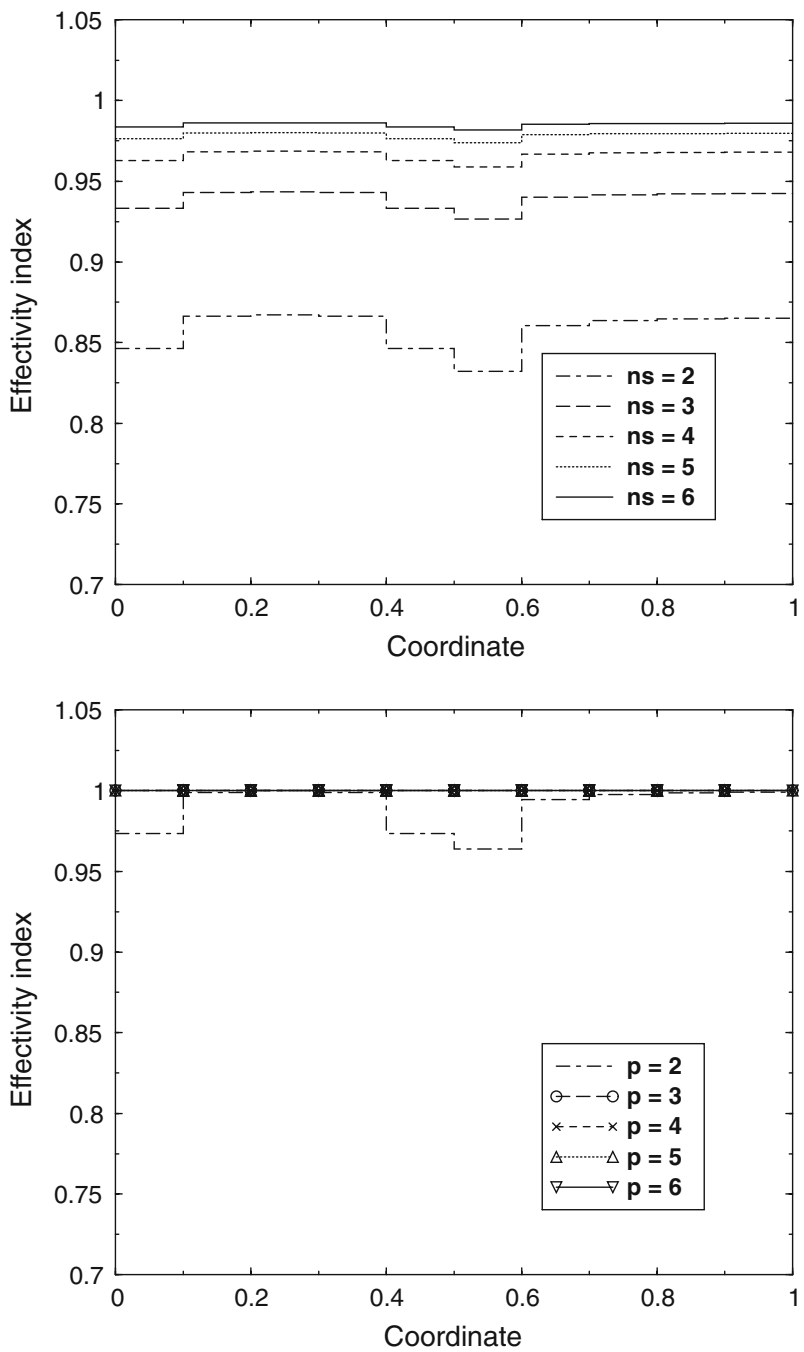

Fig. 8 Distribution of local effectivity index in the 1D problem with a ten-element discretisation, based on $h$-version (top) and $p$-version (bottom) error estimates and varying order of local refinement from 2 to 6.The number of subelements is denoted as " $n s "$

using this example is to see how good the estimator is, in estimating the interior residuals.

In Fig. 8, the local reference discretisation is varied from order 2 to order 6 . Based on $h$-extension, this means that the patch is subdivided into 2 to 6 subelements. On the other hand, in the $p$-scheme, the order of interpolation is varied from $p=2$ (quadratic) to $p=6$ (hexic) interpolations. The quality of the estimates is measured through an effectivity index, which is defined as

$\theta_{e}:=\frac{\left\|\boldsymbol{e}_{(\tilde{h}, \tilde{p})}\right\|}{\|\boldsymbol{e}\|}$

i.e. the ratio of the estimated and the exact error, $e:=u-$ $u_{(h, p)}$, measured in the energy norm.

In Fig. 8, the high effectivity index in all elements in the local $p$-version, especially when the local enrichment order

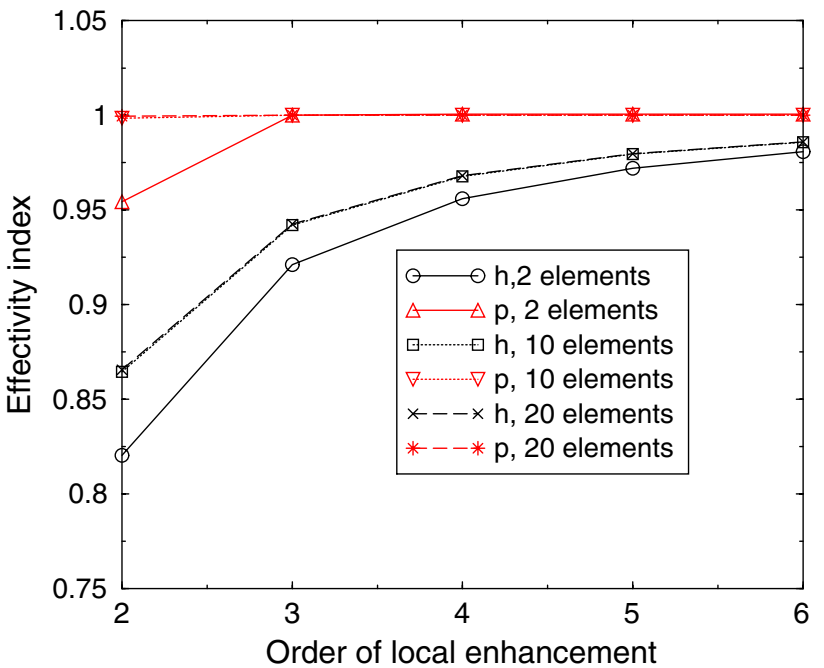

Fig. 9 The global effectivity index obtained by different orders of $\mathrm{h}$ and p-local refinement schemes in the $1 \mathrm{D}$ example with 2-element, 10element and 20-element discretisations

is higher than two, reveals that the local $p$-version performs better than the local $h$-version. This is partly due to the fact that the exact solution is in the polynomial form and thus the higher-order polynomial interpolation can capture the solution better than the element subdivision. It is shown in Fig. 9 that the estimators perform better in the more refined finite element discretisation as, at the same degree of local refinement, the local reference discretisation provides a closer representation of the exact solution.

One can notice that in Fig. 10, where we use an odd number of elements, the local effectivity index appearing in the middle elements shows how poorly the estimation performs, in both $h$-version and $p$-version, especially when applying the second order of local refinement. The improvement in effectivity index when going to third order local refinement is significant. Moreover, Fig. 11 reveals that the error estimate of the middle element can become worse as the number of degrees of freedom increases. We observe that, at the middle point (i.e. $x=0.5$ ), the curvature of the solution function changes from being convex to being concave and that should be the main reason for the poor error estimates in the element including such a transition point. Apparently, the smaller the middle element is, the bigger the relative effect of the curve transition is on the error estimate. However, the failure in the local error estimation does not affect the global measurement significantly.

\subsection{Quadratic stress test}

The error estimation is now applied to a two-dimensional problem domain. The quadratic stress patch is modelled as in Fig. 12 (left). A patch of $1 \mathrm{~mm} \times 1 \mathrm{~mm}$ is modelled in a plane stress condition with Young's modulus $E=1$ 

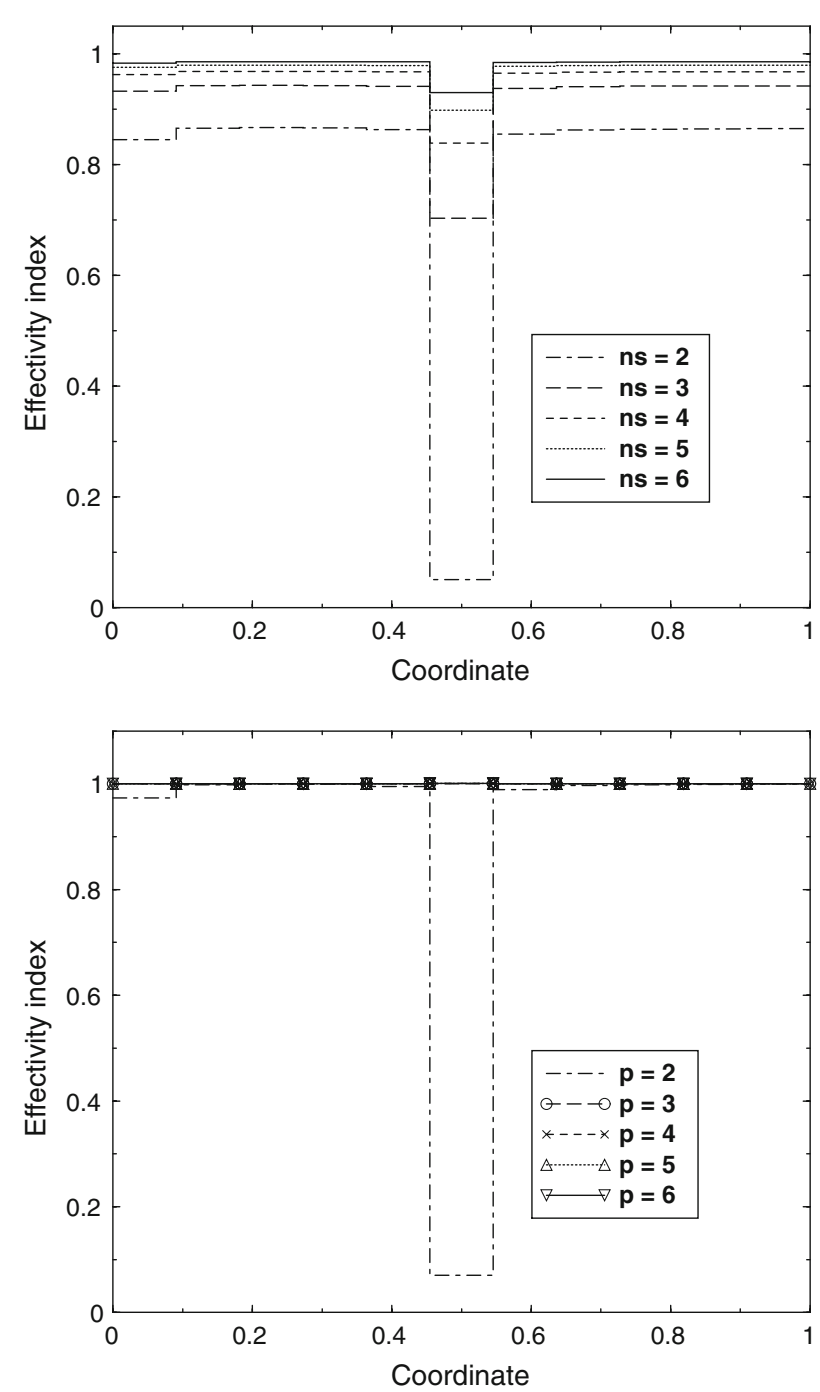

Fig. 10 Distribution of local effectivity index in the 1D problem with an 11-element discretisation, based on $h$-version (top) and $p$-version (bottom) error estimates and varying order of local refinement from 2 to 6

MPa and Poisson's ratio $v=0.25$. We model this problem by setting the non-homogeneous Dirichlet conditions of the exact solution

$u_{x}(x, y)=\frac{x^{2} y}{2 E}+\frac{v y^{3}}{6 E}-\frac{(1+v) y^{3}}{3 E}$

$u_{y}(x, y)=-\frac{v x y^{2}}{2 E}-\frac{x^{3}}{6 E}$

on the whole boundary. The finite element solution is obtained by linear interpolation.

As our error estimation scheme is originated from [7], which is based on the local $h$-extension scheme, we start our investigation with this original scheme. The error functions plotted in Fig. 13 apparently depend on how the patches overlap in the local $h$-refinement scheme [7]. In terms of performance, Fig. 14 (top) reveals a better estimation and effectivity in case of all local $p$-enrichments. In this example, a

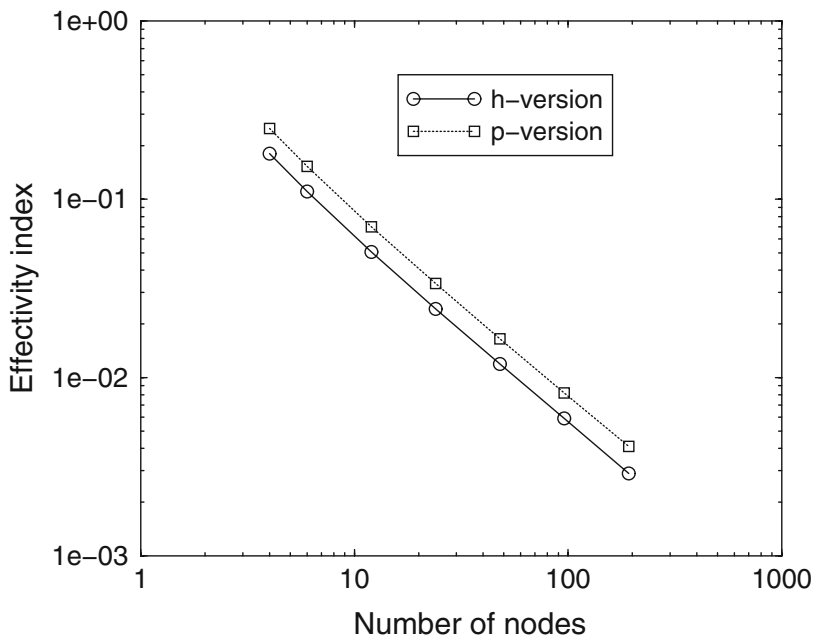

Fig. 11 Failure of the error estimates due to inadequate representation (i.e. local refinement of order 2) of the actual solutions in the 1D problem modelled by an odd number of elements. The effectivity indices shown are those of the middle elements only
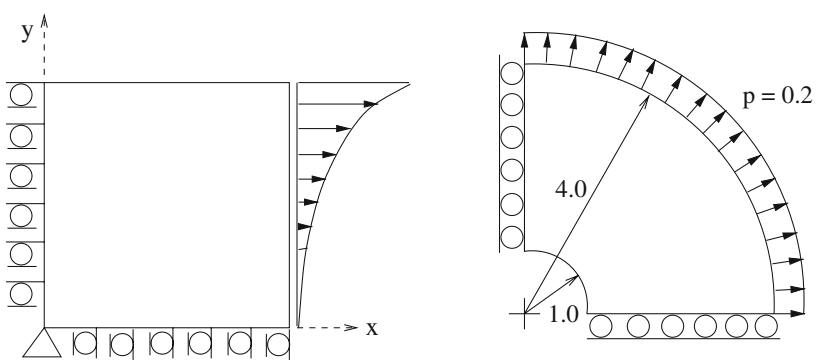

Fig. 12 The patch test (left) and the disk test (right)

triangular element is subdivided into 27 subelements, adding 68 degrees of freedom for the elemental computation in the $h$-version. Conversely, a set of quadratic polynomials is added to each element in the $p$-scheme, adding 3 degrees of freedom per element. If considering a patch of maximum six elements, we need to solve only 38 equations in each local computation. Yet, the global error estimate based on the local $p$-enrichment, in comparison to the local $h$-refinement, shows a closer estimation to the exact error computed based on the analytical solution in Eqs. 31 and 32.

The quadratic enrichment is, of course, not the most accurate choice for estimating the error in linear elements. It is, however, sufficient since the errors are much smaller in comparison to the original linear interpolation. Furthermore, enriching to a higher-order polynomial does not provide a great improvement to the error estimation, considering the fact that the number of local equations is greatly increased. However, it still provides the possibility to set the local reference solutions from higher-order polynomials, if highly accurate error solutions are necessary. In Fig. 14 (bottom), the global error estimates based on different orders of interpolation are compared. 


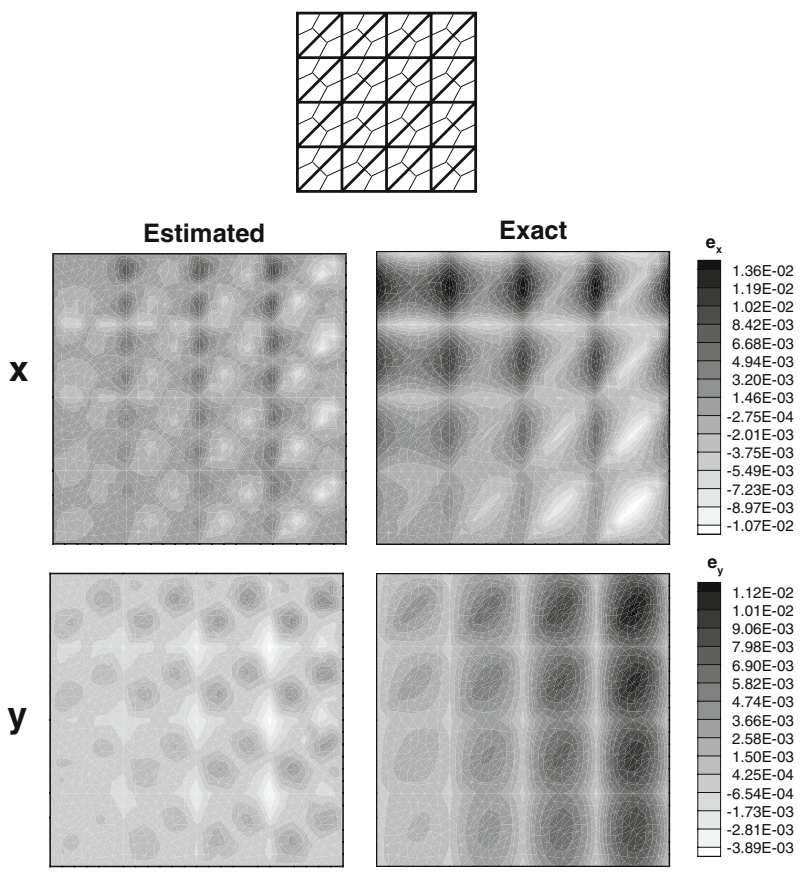

Fig. 13 The estimated and exact error functions in $x$ - and $y$-directions of the quadratic stress test. The mesh (thick line) and the overlapping patches (thin line) are shown at the top

Before moving further to investigate the performance of error estimation in higher-order elements, let us consider the actual error trend of the quadratic stress test, as shown in Fig. 15. Evidently, the exact solution can be obtained by applying cubic interpolation in the finite element modelling, but not by the same number of equations in the element subdivision scheme. Comparing the same number of degrees of freedom, the polynomial enrichment shows a more efficient trend. For this reason, the local $p$-enrichment scheme is more efficient than the local $h$-subdivision. However, if cubic polynomials, which represent the exact solution, are employed, why can the estimator not exactly predict the actual error? [See Fig. 14 (bottom).] To come up with an explanation, we refer back to the basic concept in Sect. 4.2, i.e. the error is prescribed as zero on $\partial \Omega_{k} \backslash\left(\partial \Omega_{k} \cap \Gamma_{n}\right)$. This means, the error on the boundary, which is linearly interpolated, is assumed null, while the real error should in fact be cubically interpolated. A good proof for this point is shown by the local error plot in Fig. 16, where the estimated and the actual error functions along the boundary differ. Based on this interpretation, the estimator can never recover the exact error unless the primary solution on the boundary contains the polynomial order that interpolates the exact solution.

We investigate further the error estimate in higher-order elements. Following the same strategy, a set of higher-order terms is added for each displacement mode. As mentioned earlier, for each polynomial order of the displacements, the error is estimated with one higher polynomial order. The
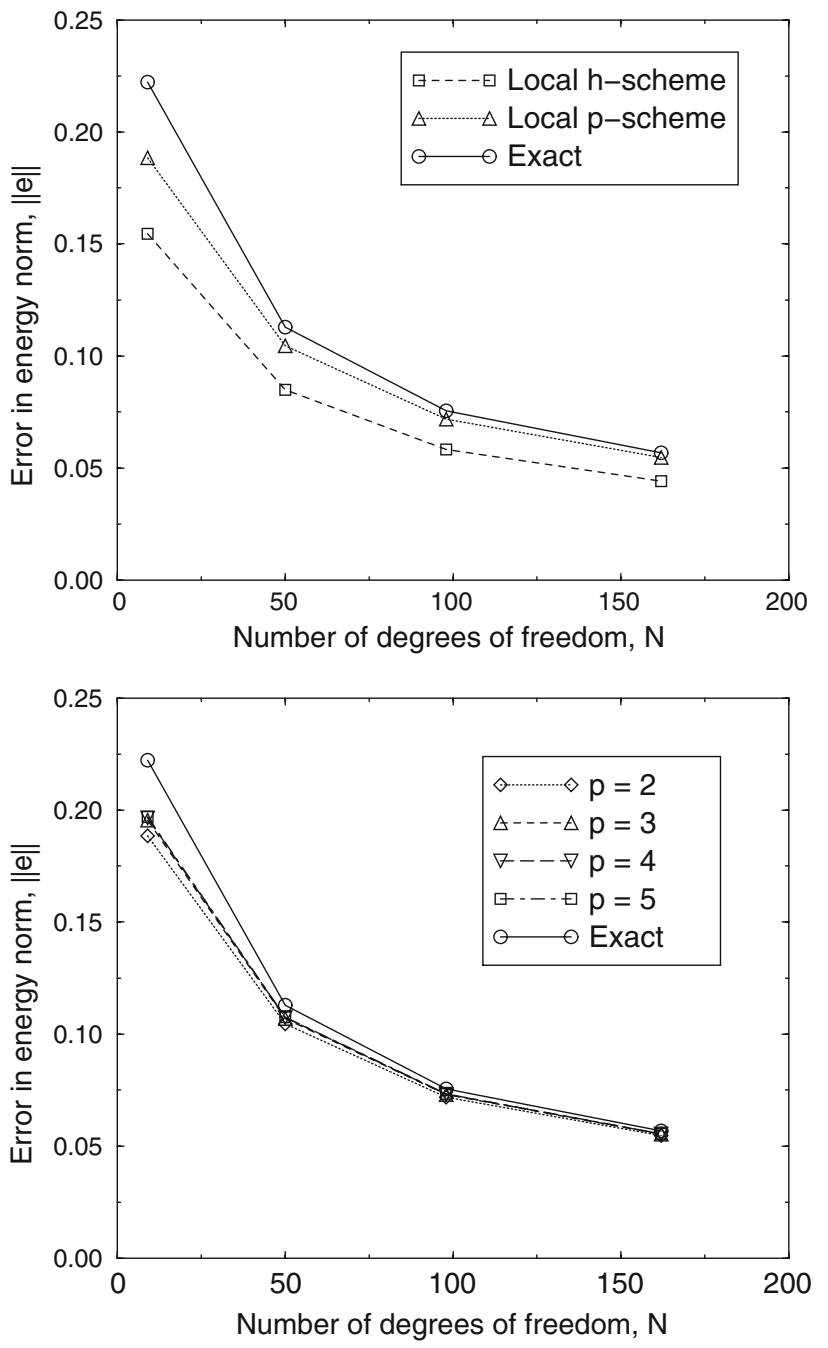

Fig. 14 Comparison of estimated error in energy norm by different local enrichment schemes in the quadratic stress test

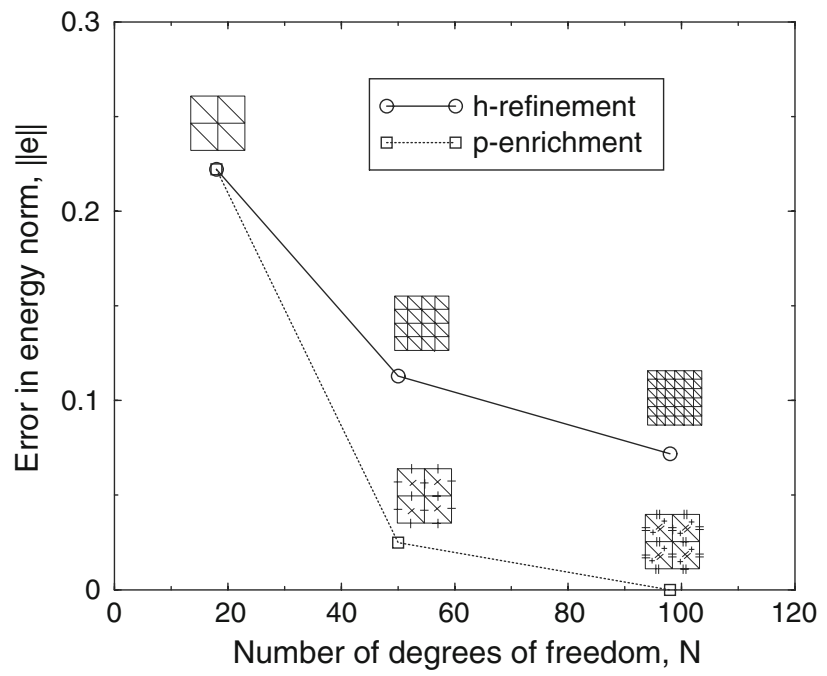

Fig. 15 Convergence analysis of quadratic stress patch problem based on global $h$-refinement and global $p$-enrichment 

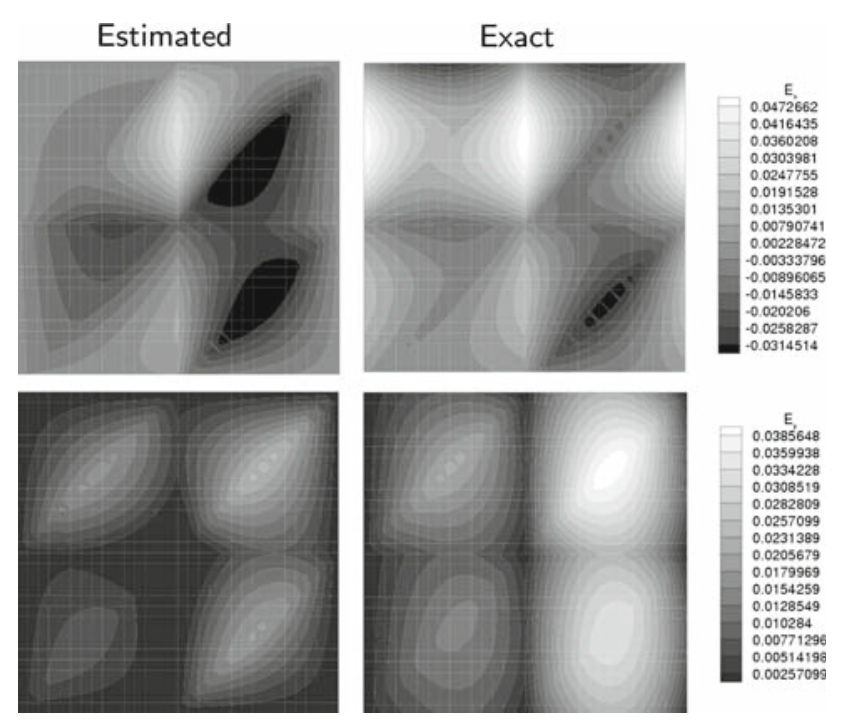

Fig. 16 Comparison between the estimated error function (left) and the exact error function (right), based on analytical solutions, in the $x$ (top) and $y$ - (bottom) direction of the linearly interpolated patch

Table 2 Global error in energy norm obtained in quadratic stress patch test. Dirichlet boundary conditions are imposed by direct imposition scheme in the standard $p$-finite element modelling

\begin{tabular}{llllll}
\hline Model & DOFs & Reference & $\|\boldsymbol{e}\|$ & $\|\boldsymbol{e}\|^{\mathrm{ex}}$ & $\theta_{e}(\%)$ \\
\hline$p=1$ & 18 & $p=2$ & 0.1885 & 0.2223 & 84.8 \\
$p=2$ & 50 & $p=3$ & 0.0522 & 0.1176 & 44.4 \\
$p=3$ & 98 & $p=4$ & 0.0199 & 0.1051 & 18.9 \\
\hline
\end{tabular}

quadratic stress patch is again tested and the results are shown in Table 2, where the global performance is measured in terms of the effectivity index. As shown, the effectivity index is greatly reduced when the element polynomial order increases. This is not a surprising phenomenon in this problem, which consists of non-homogeneous Dirichlet boundary conditions. Since there are no additional nodes in the hierarchical $p$-extension, prescribing the boundary conditions implies only a linear variation of displacements at the boundary. Without any special techniques, it is rather difficult to directly impose the exact displacements, which are higherorder polynomials, along the boundary. The higher-order polynomials along the boundary edges are dropped out and the global boundary conditions are, instead, represented by linear interpolation. Obviously, the reference model, in this case, cannot resemble the actual model, thus leading to failure of the error estimation.

If the prescribed displacements are imposed exactly, we should be able to obtain a good error estimate in the patch problem. The proper enforcement of boundary conditions is made possible by, e.g. adding Lagrange multipliers or penalty terms in the variational formulation. As a result, Eq. 2 is replaced by
$\mathcal{B}^{*}\left(\boldsymbol{u}_{(h, p)}, \boldsymbol{v}_{(h, p)}\right)=\mathcal{L}\left(\boldsymbol{v}_{(h, p)}\right) \quad \forall \boldsymbol{v}_{(h, p)} \in \mathcal{V}_{(h, p)}$

where $\mathcal{B}^{*}(\cdot, \cdot)$ is equivalent to the original $\mathcal{B}(\cdot, \cdot)$, further including the already prescribed Dirichlet boundary conditions. The displacements on the boundary edges can then be properly imposed. As a consequence, the error estimation with penalty functions provides a better estimate than the error estimation with the direct imposition model, as shown in Table 3. The errors are better estimated in the higher-order elements because, firstly, the reference model contains a better solution, i.e. the reference error becomes closer to zero. Secondly, the errors on the global Dirichlet boundary become closer to zero, thus closer to the basic assumption of this error estimation.

Figures 16 and 17 present the local error distribution for the eight-element patch. The comparison between the estimated errors (left) and the actual errors (right) yields very similar profiles, especially in the case of higher-order elements. It can be seen that, since the displacements are prescribed along the whole domain, the errors are forced to zero. This, of course, affects the error distributions in the boundary zones. The error contour in the case of quadratic elements in Fig. 17 apparently reveals a closer estimate to the actual error

Table 3 Global error in energy norm obtained in quadratic stress patch test. Dirichlet boundary conditions are imposed by penalty formulation in the standard $p$-finite element modelling

\begin{tabular}{llllll}
\hline Model & DOFs & Reference & $\|\boldsymbol{e}\|$ & $\|\boldsymbol{e}\|^{\mathrm{ex}}$ & $\theta_{e}(\%)$ \\
\hline$p=1$ & 18 & $p=2$ & 0.1885 & 0.2223 & 84.8 \\
$p=2$ & 50 & $p=3$ & 0.0234 & 0.0249 & 94.0 \\
$p=3$ & 98 & $p=4$ & 0.0000 & 0.0000 & 100.0 \\
\hline
\end{tabular}
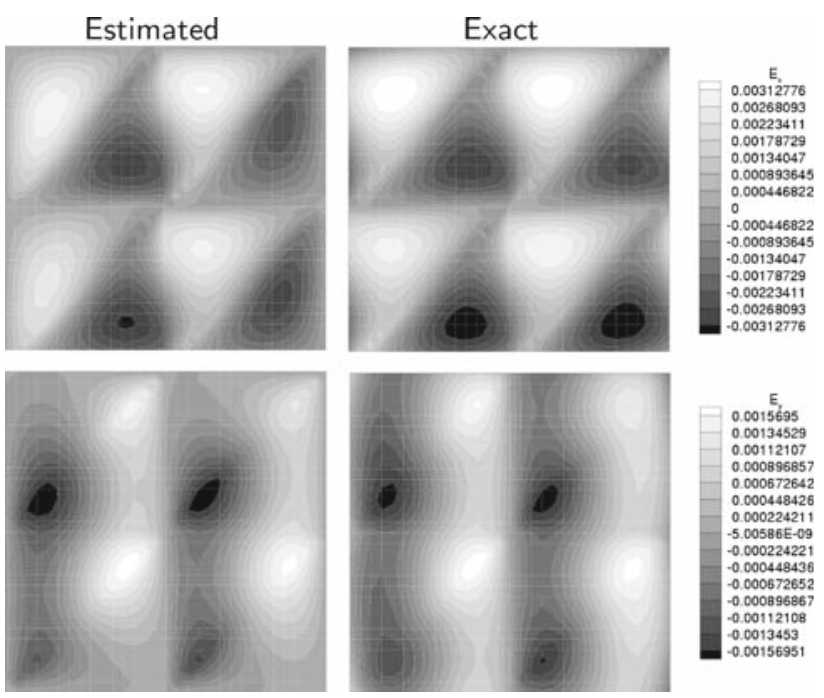

Fig. 17 Comparison between the estimated error function (left) and the exact error function (right), based on analytical solutions, in the $x$-(top) and $y$-(bottom) direction of the quadratically interpolated patch 
distribution. Even better, the estimate in the cubic elements shows zero error in the whole domain, which is the same as the exact error plot.

\subsection{Disk problem}

Finally, consider a disk being pulled uniformly at the outer boundary, as shown in Fig. 12 (right). We neglect the discretisation error due to the geometrical modelling by assuming that the disk is not circular but composed of linear boundary segments. In this example, we would like to study the effect of the missing global part in local error estimation scheme. To this end, we compare the estimated error $\left(\|\boldsymbol{e}\|^{\text {loc }}\right)$ and the error obtained by the difference between two sets of global finite element solutions, the original and the reference, $\left(\|\boldsymbol{e}\|^{\text {glob }}\right)$, defined as

$\|\boldsymbol{e}\|^{\mathrm{glob}}=\left[\int_{\Omega}\left(\boldsymbol{\varepsilon}_{(p+1)}-\boldsymbol{\varepsilon}_{(p)}\right)^{T}: \boldsymbol{D}:\left(\boldsymbol{\varepsilon}_{(p+1)}-\boldsymbol{\varepsilon}_{(p)}\right) \mathrm{d} \Omega\right]^{\frac{1}{2}}$

where $\boldsymbol{\varepsilon}_{(p)}$ and $\boldsymbol{\varepsilon}_{(p+1)}$ represent the set of strain components computed using $(p)$-order and $(p+1)$-order interpolation, respectively. In other words, Eq. 34 provides the error computed via the global stiffness matrix, and this reference error is used to calibrate the locally computed error estimate.

The global results in Table 4 exhibit good agreement between the errors based on the local computation $\|\boldsymbol{e}\|^{\text {loc }}$ and those based on the global computation $\|\boldsymbol{e}\|^{\text {glob }}$, especially in the case with higher-order elements. This suggests that, in this problem, error solutions from a coarse mesh may be sufficiently recovered by the local computation, which is definitely much cheaper than the global computation underlying Eq. 34. Also, this implies that the missing global part is of a marginal magnitude. The local contour plots in Fig. 18 again show a good agreement.

It should be noted here that, in order to obtain a good error estimate, a refined local patch must resemble the (reference) refined global system as much as possible. For example, a circular disk can be modelled by either prescribing displacements or prescribing forces along its outer boundary. The displacement control model (Fig. 19b) provides different

Table 4 Global error in energy norm obtained in circular disk problem

\begin{tabular}{lclll}
\hline Model & DOFs & Reference & $\|\boldsymbol{e}\|^{\text {loc }}$ & $\|\boldsymbol{e}\|^{\text {glob }}$ \\
\hline$p=1$ & 50 & $p=2$ & 0.0872 & 0.0897 \\
$p=2$ & 162 & $p=3$ & 0.0176 & 0.0176 \\
$p=3$ & 338 & $p=4$ & 0.0101 & 0.0101 \\
$p=4$ & 578 & $p=5$ & 0.0087 & 0.0087 \\
\hline
\end{tabular}
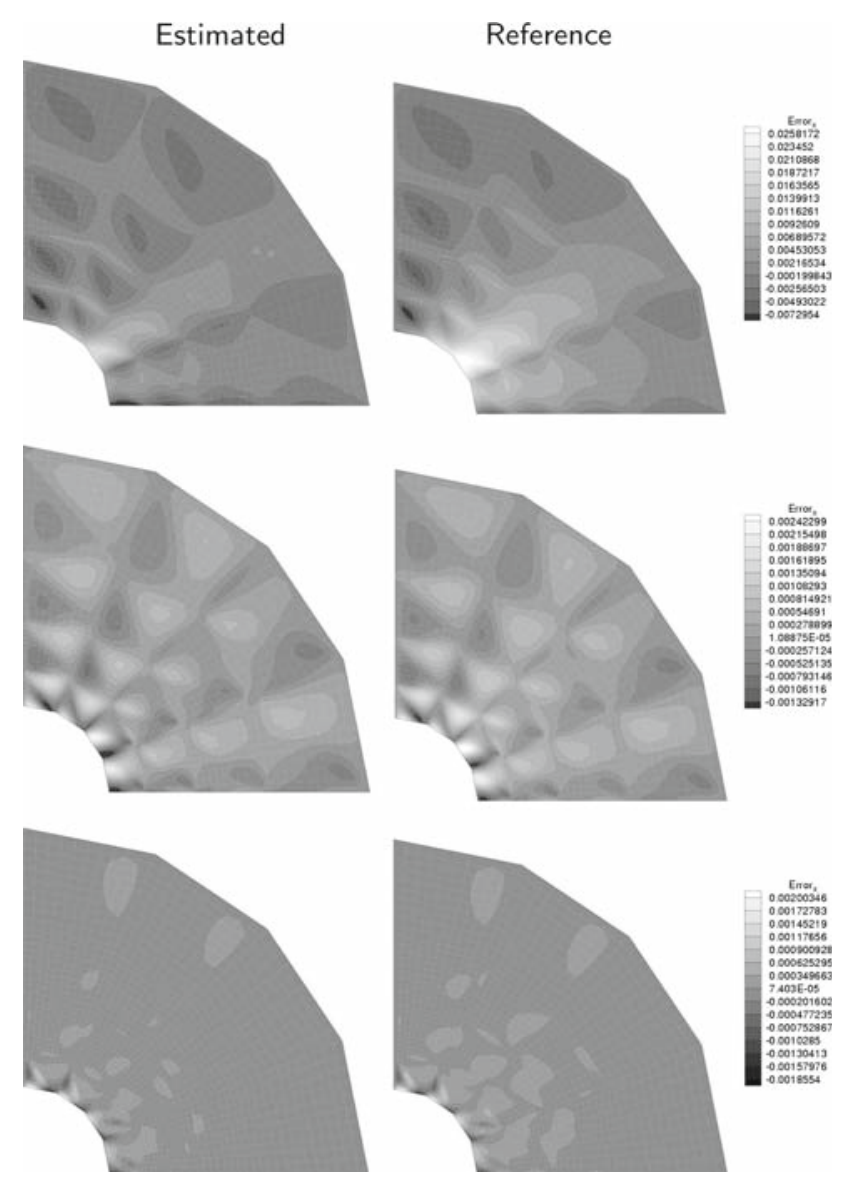

Fig. 18 Comparison between the estimated error function (left) and the reference error function (right), based on solutions from a higherorder interpolation scheme, in the $x$-direction of the circular disk. The polynomial basis is varied from $p=1$ to $p=3$ as shown in uppermost to lowermost subfigures

error patterns than the force control model (Fig. 19C), since we assume exact displacements along the global Dirichlet boundary, i.e. $e(\mathbf{x})=0, \forall \mathbf{x} \in \Gamma_{d}$. This means, displacements or forces must be correctly prescribed on the local boundary and must resemble the real globally refined model. In this example, if one fails to correctly prescribe the interpolated forces along the outer boundary, instead of Fig. 19c, an obviously wrong estimation can be obtained, as shown in Fig. 19d.

\section{Concluding remarks}

In this article, a $p$-version error estimator has been proposed. The method, based on enhancing each local domain with a higher-order polynomial interpolation, has shown a good performance in estimating the error. Compared to the earlier formulated $h$-version, the $p$-version can provide a more efficient error estimate; a smaller system of local equations is used to obtain the same accuracy. Even then, the quality of the error estimate depends on the finite element discre- 


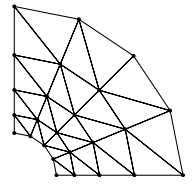

(A)

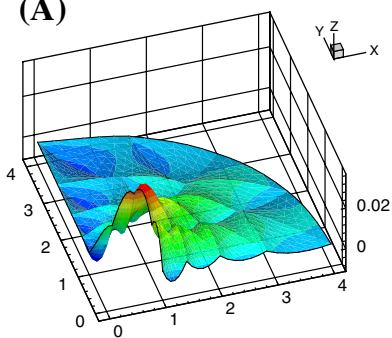

(B)

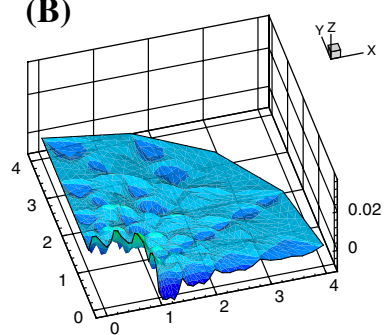

(C)
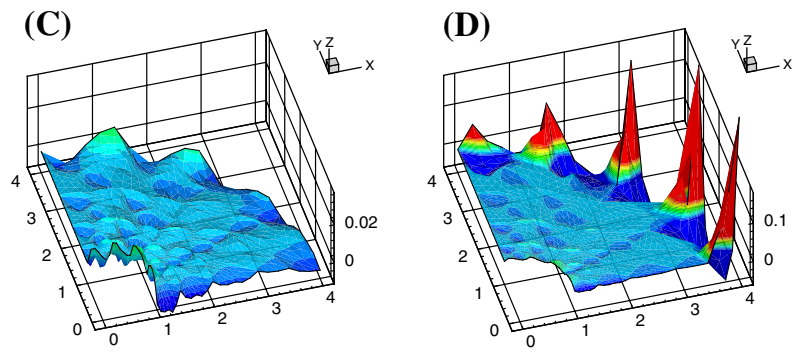

Fig. 19 Functions of error in $x$-displacement of the circular disk problem: a expected error, $\mathbf{b}$ estimated error in the prescribed displacement model, c estimated error based on the prescribed force model and $\mathbf{d}$ estimated error based on prescribed force model (improper modelling)

tisation. The error estimate of the element where there is a change of the solution curvature can locally be poor, and a sufficiently-high order of local refinement/enrichment should be applied. It has also been found that the estimate is very sensitive to the choice of the reference mesh and, in particular, the description of the boundary conditions. Without a correct reference discretisation, the estimated error can be far from the exact error.

The main purpose of this study is to investigate the performance of the $p$-version error estimation as an alternative to the original $h$-version. In this study, we have not investigated the cases in which the regularity of the exact solution is low. In those case, it is possible that adding more degrees of polynomials in those regions does not always guarantee a more accurate solution. However, we have observed in elsewhere $[11,14]$ that, the estimated error in such regions (the crack tip region, for example) is still dominant in comparison to other regions. It is then recommended to combine the error estimation with the adaptive techniques such as $h$-adaptivity or $r$-adaptivity, which helps capturing a low regularity solution. Upon those mesh improvement processes, the estimated error in the problem region will gradually be improved.

Open Access This article is distributed under the terms of the Creative Commons Attribution Noncommercial License which permits any noncommercial use, distribution, and reproduction in any medium, provided the original author(s) and source are credited.

\section{References}

1. Ainsworth M (1994) The performance of Bank-Weiser's error estimator for quadrilateral finite elements. Numer Methods Partial Differ Equ 10:609-623

2. Ainsworth M (1996) The influence and selection of subspaces for a posteriori error estimators. Numer Math 73:399-418

3. Ainsworth M, Oden JT (1993) A unified approach to a posteriori error estimation based on element residual methods. Numer Math $65: 23-50$

4. Ainsworth M, Oden JT (2000) A posteriori error estimation in finite element analysis. Wiley, New York

5. Babuška I, Rheinboldt C (1978) A-posteriori error estimates for the finite element method. Int J Numer Methods Eng 12:1597-1615

6. Bank RE, Weiser A (1985) Some a posteriori error estimators for elliptic partial differential equations. Math Comput 44:283-301

7. Díez P, Egozcue JJ, Huerta A (1998) A posteriori error estimation for standard finite element analysis. Comput Methods Appl Mech Eng 163:141-157

8. Díez P, Arroyo M, Huerta A (2000) Adaptivity based on error estimation for viscoplastic softening materials. Mech Cohesive Frictional Mater 5:87-112

9. Huerta A, Díez P (2000) Error estimation including pollution assessment for nonlinear finite element analysis. Comput Methods Appl Mech Eng 181:21-41

10. Ladevèze P, Leguillon D (1983) Error estimate procedure in the finite element method and applications. SIAM J Numer Anal 20:485-509

11. Pannachet T (2006) Error estimation and adaptive spatial discretisation for quasi-brittle failure. $\mathrm{PhD}$ thesis, Delft University of Technology, The Netherlands

12. Pannachet T, Askes H (2002) Partition-of-unity based p-adaptivity and error estimation in the finite element method. In: WCCM $\mathrm{V}$-fifth world congress on computational mechanics, Vienna, Austria

13. Pannachet T, Askes H (2003) A residual-type error estimate for $p$-version finite element method. In: Proceedings of international conference on adaptive modeling and simulation (ADMOS 2003), Gothenburg, Sweden

14. Pannachet T, Sluys LJ, Askes H (2008) Error estimation and adaptivity for discontinuous failure. Int J Numer Methods Eng (submitted)

15. Rodríguez-Ferran A, Huerta A (2000) Error estimation and adaptivity for non-local damage models. Int J Solids Struct 37:7501-7528

16. Szabó B, Babuška I (1991) Finite element analysis. Wiley, New York

17. Taylor RL, Zienkiewicz OC, Oñate E (1998) A hierarchical finite element method based on partition of unity. Comput Methods Appl Mech Eng 152:73-84

18. Verfürth R (1996) A review of a posteriori error estimation and adaptive mesh refinement techniques. Wiley-Teubner, New York

19. Zienkiewicz OC, Taylor RL (1994) The finite element method. Basic formulation and linear programs, vol 1, 4th edn. McGrawHill, New York

20. Zienkiewicz OC, Zhu JZ (1987) A simple error estimator and adaptive procedure for practical engineering analysis. Int $\mathrm{J}$ Numer Methods Eng 24:337-357

21. Zienkiewicz OC, Zhu JZ (1992) The superconvergent patch recovery (SPR) and adaptive finite element refinement. Comput Methods Appl Mech Eng 101:207-224 